\title{
Pore-scale lattice Boltzmann simulation of micro-gaseous flow considering surface diffusion effect
}

\author{
Junjian Wang ${ }^{\mathrm{a}}$, Qinjun Kang ${ }^{\mathrm{b}}$, Li Chen ${ }^{\mathrm{b}, \mathrm{c}}$, Sheik S Rahman ${ }^{\mathrm{a}, *}$ \\ ${ }^{a}$ School of Petroleum Engineering, University of New South Wales, Sydney, NSW,Australia,2033 \\ ${ }^{b}$ Earth and Environmental Sciences Division, Los Alamos National Laboratory, Los Alamos, NM,USA,87545 \\ ${ }^{c}$ Key Laboratory of Thermo-Fluid Science and Engineering of MOE, School of Energy and Power Engineering, Xi'an \\ Jiaotong University, Xi'an, Shanxi, China, 710049
}

\begin{abstract}
Recent studies have shown that adsorbed gas and its surface diffusion have profound influence on micro-gaseous flow through organic pores in shale gas reservoirs. In this paper, a multiple-relaxation-time (MRT) LB model is adopted to estimate the apparent permeability of organic shale and a new boundary condition, which combines Langmuir adsorption theory with Maxwellian diffusive reflection boundary condition, is proposed to capture gas slip and surface diffusion of adsorbed gas. The simulation results match well with previous studies carried out using Molecular Dynamics (MD) and show that Maxwell slip boundary condition fails to characterize gas transport in the near wall region under the influence of the adsorbed gas. The total molar flux can be either enhanced or reduced depending on variations in adsorbed gas coverage and surface diffusion velocity. The effects of pore width, pressure as well as Langmuir properties on apparent permeability of methane transport in organic pores are further studied. It is found that the surface transport plays a significant role in determining the apparent permeability, and the variation of apparent permeability with pore size and pressure is affected by the adsorption and surface diffusion.
\end{abstract}

Keywords: lattice Boltzmann method, adsorbed gas, surface diffusion, micro-gaseous flow

\section{Introduction}

The emerging development of the unconventional gas reservoirs such as tight gas, coal seam gas and shale gas reservoir in last decades has raised the necessity for advancing our understanding of the physical laws governing the gas flow in nano-/micro- pores. In these reservoirs, the matrix pore space can be on the same order of magnitude as the mean free path of the gas molecules. According to the Knudsen number $(K n)$ and Mach number $(M)$, the dynamics of nano-/micro- scale gas flow can be similar to that of rarefied gas flows[1]. Based on this assumption, a variety of slip models such as the extended slip N-S model[2] and empirical slip models $[3,4]$ were developed and widely applied to study gas transport in unconventional reservoirs. However, at reservoir conditions, the presence of a large amount of adsorbed gas is also

${ }^{*}$ Corresponding author at: School of Petroleum Engineering, UNSW, Sydney, NSW, Australia, 2033

Email address: sheik.rahman@unsw.edu.au (Sheik S Rahman)

Preprint submitted to Elsevier

August 7, 2016 
very common in industrial applications especially in organic shale gas reservoir[5, 6], and the impact of surface diffusion of adsorbed gas may be significant on the gas transport process[7]. Consequently, some phenomenological models in a superposition form of surface diffusion, Knudsen diffusion, slip flow and viscous flow were proposed[8, 9, 10], and all of them conclude a fast mass transport of gas at nano- to micro-scale depending on Knudsen number and surface diffusivity. While this conclusion was adopted from methane flow in graphitic and the different potentials between gas and substrates[11] are ignored, an emerging number of molecular dynamics (MD) studies[1, 11, 12, 13] on different gas-surface interaction potentials have been carried out and they claimed the deficiency of previous slip and superposition models in characterizing the micro-gas flow in a confined system with an adsorbed gas field. Although MD provides the ultimate level of details of individual particles/molecules, its huge cost on account of the vast number of molecules required to simulate limits its further applications to a large simulation domain. In recent years, lattice Boltzmann method (LBM), a mesoscopic simulation approach, by combining aspects of particle-based (microscopic) and continuum-based (macroscopic) methods, renders it ideally suited to modelling microfluidics and micro-gaseous flow in unconventional reservoirs [14, 15].

Recently, LB algorithms were developed to characterize slip effect in shale at both representative elementary volume (REV)-scale and pore-scale. Chen et al.[16, 17] cite extended the generalised LBM for slip flow at REV-scale, in which Beskok and Karniadakis-Civan's correlation[4] was used to calculate the local apparent permeability. Chen and coauthors also developed a porescale LBM [18, 19] based on the Dusty gas model (DGM), which is a superposition of viscous flow and Knudsen diffusion, to estimate the apparent permeability and the Knudsen diffusivity of reconstructed organic shale, and their results match well with previous studies. Still the slip approach is a popular choice, and a variety of slip boundary conditions as a function of the tangential momentum accommodation coefficient (TMAC) such as the Maxwellian diffusive boundary condition[20], combined bounce back with specular reflection boundary condition (CBBSR)[21] or combined bounce back with diffusive reflection boundary condition (CBBDR)[22] were developed, and with these boundary conditions LBM can be recognized as an efficient alternative to solve Navier-Stocks (N-S) equation in tackling rarefied gas flow problems.

Most recently, a few attempts were made in literature to apply LBM to simulate microgaseous flow in kerogen/organic matter by considering the gas adsorption and/or surface diffusion effects. Wang et al.[23] estimated the apparent permeability of organic shale with the use of a generalised LBM, in which the surface diffusion is considered with the aid of DGMgeneralised Maxwell Stefan (GMS) model. In order to account for the adsorbed gas effect, Allan and Mavko[24] as well as Li et al.[25] introduced the adsorbed gas as an immobile layer to organic pores which decreases the permeability by reducing pore space. Allan and Mavko[24] also claimed that the adsorption layer will change the TMAC, but this claim was not substantiated in their study. Fathi and Akkutlu [26] used Shan-Chen model to simulate gas flow in shale. The authors also used Langmuir slip boundary condition as proposed by Chen and Tian[27] to consider the interaction forces on the solid surfaces. The simulation results show that, when surface diffusion coefficient is larger than molecular diffusion coefficient at high pressure or when the gas flows in a capillary less than $20 \mathrm{~nm}$ at low pressure, the fluid velocity at the wall can exceed the fluid velocity at the centre of the capillary. Later, both Ren et al.[10] and Li et al.[25] argued that the combination of Shan-Chen model and the Langmuir boundary condition might double count the gas-solid interactions, which could introduce some non-physical phenomena in simulations. In order to overcome the discrete effect, Ren et al.[10] treated the surface diffusion of adsorbed gas as a moving wall in a CBBSR boundary condition with the premise that the gas in bulk flow 
is assumed to "slip" above the adsorption layer. However, the exchange of adsorbed gas and free gas resulting from the dynamic adsorption/desorption is not considered in their model.

Based on the above review, the application of LBM to gas flow at micro-scale is still in its infancy. Although many LB models have been proposed to capture adsorbed gas and/or surface diffusion, they are controversial to each other because the lack of complete knowledge of surface properties and surface interaction potentials. In this current study, we focus on the gassurface interaction and we aim to present a new boundary condition for the LB model to couple the adsorbed gas and surface diffusion effects based on previous MD simulation observations. The paper is organized as follows: The physical representation and mathematical formulation of LBM with the new boundary condition are introduced in sections 2 and 3. The validation of numerical model is presented in section 4. Finally, in section 5, the role of adsorbed gas coverage and surface diffusion velocity on gas flow behaviour at micro-scale are discussed, and then a particular application to organic shale is studied to analyse the effects of pore width, pressure and Langmuir parameters on gas apparent permeability.

\section{Inter-facial physics of gas-solid interaction}

With the decrease of length scale, the ratio of surface to volume increases rapidly and surface forces become dominant. Surface-fluid interaction governs momentum and energy transport and the gas molecular kinetics are affected by the wall molecules. When a gas molecule comes into contact with the solid surface, it may experience various events such as elastic scattering, inelastic scattering, temporary or permanent adsorption, and mobile adsorption (surface diffusion)[28]. Within the kinetic theory framework of the Maxwell slip velocity model[29], the scattering of particles was classified as specular reflection and diffusive reflection. In the specular reflection model, the sticking time is virtually negligible and incident molecules undergo perfect elastic collision with the wall, such that there is no exchange of energy with the surface and the reflected molecules have the same velocity as that of the incident molecules. In the diffuse reflection model, incident molecules reside on the wall for a long time, during which it interacts with solid atoms and completely thermalized with the surface before scattering back, such that the net velocity of reflected molecules will be the same as that of the wall (see Fig. 1). While the Maxwell model and subsequent extensions of the model have shown moderate success in the prediction of flows on smooth surfaces or rarefied gas flow with extremely long adsorption time[30], some MD simulation studies[31, 32, 33, 34] argue their deficiency in capturing complete physics associated with complex problems such as short time adsorption/desorption-related effects and surface roughness effects. 


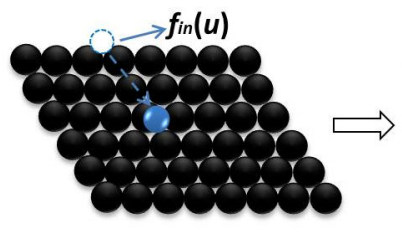

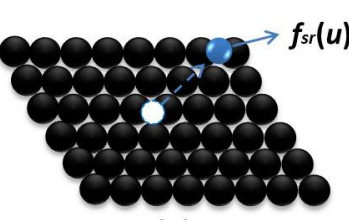

(a)

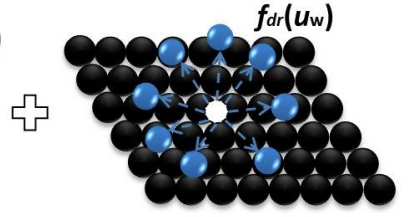

(b)

Figure 1: Schematic of gas-solid interactions without considering moderate adsorption/desorption and surface diffusion based on Maxwell slip theory[29]. $f_{\text {in }}$ represents incident molecules with a velocity $\mathbf{u}$; in sub-figure $(a), f_{s r}$ represents specularly reflected molecules with the same velocity as that of incident molecules after undergoing perfect elastic collision with the wall; in sub-figure $(b), f_{d r}$ represents diffusively reflected fluid molecules with the same velocity as that of the solid wall after the incident molecules adsorbed near the wall for a long time and totally forget the incident information.

When gas molecules are easy to be adsorbed near the wall under strong physical interactions, and in a situation where the potential energy barrier to diffusion is smaller than the energy of desorption for diffusion to occur, an adsorbed particle may remain mobile in a metastable phase while still being physically bound to the surface. This phenomenon is known as the surface diffusion[35] (see the movement of red gas molecules along the surface in Fig. 2). Besides, there is also a probability that adsorbed molecules may escape from the surface before reaching thermal equilibrium with the surface after a short time, and leave prematurely at a velocity $\mathbf{u}_{d e s}$ with a portion of its energy dissipated (see Fig. 2(c))[36]. Therefore, after the incident gas molecules collide with the solid wall, in addition to the reflected behaviours that could be expressed by Maxwell slip model, they would also be adsorbed on the surface and move in terms of surface diffusion or escape back into the bulk gas before reaching thermal equilibrium with the surface. (see Fig. 2(c)).

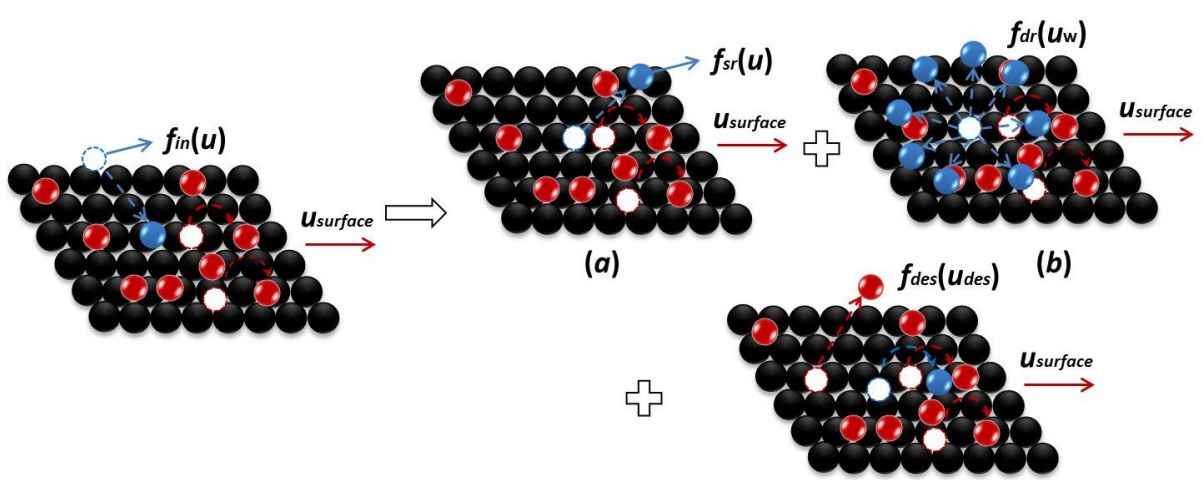

(c)

Figure 2: Schematic of gas-solid interactions considering surface diffusion and adsorption/desorption based on Maxwell slip theory[29] and inter-facial physics with adsorption field[35, 36]. Red molecules represent gas molecules being adsorbed physically on solid and moving forward with a total surface diffusion velocity $\mathbf{u}_{\text {surface }}$. $(a)$ denotes the specular reflected gas molecules, $(b)$ denotes the diffusive reflected gas molecules and $(c)$ denotes the adsorption of incident gas molecules and the premature desorption of adsorbed gas molecules with a velocity $\mathbf{u}_{d e s}$.

A completely mathematical description of gas-surface interaction is under development. 
However, as the adsorption phase contributes to transport of gas to a great extent in some cases, many studies have been carried out in regards to this aspect. The Langmuir model[37] and the Brunauer-Emmett-Teller (BET) model[38] are widely used to model adsorbing capacity of mono-layer adsorption and multilayer adsorption, respectively. Also, surface diffusion of adsorbed gas was introduced by hopping process [35] or random walk statistics[39] to explain the mass flow in excess to that predicted by Knudsen's model. Krishna and Wesselingh[9] proposed a Maxwell-Stefan approach combined with the Langmuir adsorption isotherm (which implicitly implies equilibrium), and yielded a reasonable expression for the description of surface diffusion which is confirmed by experimental work and simulation studies[40, 41]. In their approach the molar flux of surface diffusion in a single component system can be expressed as:

$$
N_{\text {surface }}=q_{\text {sat }} \frac{D_{s}}{1-\theta} \nabla \theta,
$$

where $D_{s}$ is the Maxwell-Stefan diffusivity that can be estimated from experimental studies or molecular dynamic (MD) simulations, $q_{\text {sat }}$ is the Langmuir volume, and $\theta$ is the fractional coverage of adsorbed gas which satisfies $\theta=q / q_{s a t}=b P /(1+b P)$ based on Langmuir model, where $b$ is the Langmuir constant, $P$ is the pressure, and $q$ is the surface concentration. Therefore, the surface diffusion velocity $u_{\text {surface }}$ can be written as:

$$
u_{\text {surface }}=\frac{N_{\text {surface }} M}{\rho_{\text {ads }}}=\frac{q_{\text {sat }} M}{\rho_{\text {ads }}} \frac{b D_{s}}{1+b P} \nabla P,
$$

where $M$ and $\rho_{a d s}$ are molecular weight of adsorbed gas and the density of adsorbed gas, respectively.

\section{Lattice Boltzmann model}

\subsection{LB equation}

The multiple-relaxation-time (MRT)-LB equation derived from Boltzmann equation can be written as[42]:

$$
f_{i}\left(\mathbf{x}+\mathbf{c}_{i} \delta_{t}, t+\delta_{t}\right)-f_{i}(\mathbf{x}, t)=\Omega_{i}(f),
$$

where $f_{i}(\mathbf{x}, t)$ is the discrete distribution function for particles with velocities $\mathbf{c}_{i}$ at position $\mathbf{x}$ and time $t, \delta_{t}$ is the time step, and $\Omega_{i}(f)$ is the MRT discrete collision operator.

In D2Q9 model, the discrete velocity $\mathbf{c}_{i}$ is defined as $\mathbf{c}_{1}=(0,0), \mathbf{c}_{2}=-\mathbf{c}_{4}=(1,0) c, \mathbf{c}_{3}=$ $-\mathbf{c}_{5}=(0,1) c, \mathbf{c}_{6}=-\mathbf{c}_{8}=(1,1) c, \mathbf{c}_{7}=-\mathbf{c}_{9}=(-1,1) c$. where $c=\delta_{x} / \delta_{t}$ is the lattice speed with $\delta_{x}$ being the lattice spacing. The MRT collision operator is given by:

$$
\Omega_{i}(f)=-\left(\mathbf{M}^{-1} \mathbf{S M}\right)_{i j}\left(f_{j}-f_{j}^{e q}\right),
$$

where $\mathbf{M}$ is a orthogonal transformation matrix and $\mathbf{S}=\operatorname{diag}\left(\tau_{\rho}, \tau_{e}, \tau_{\varepsilon}, \tau_{j}, \tau_{q}, \tau_{j}, \tau_{q}, \tau_{s}, \tau_{s}\right)^{-1}$ is a diagonal matrix.

The distribution function $f_{i}$ and its equilibrium distribution function $f_{i}^{e q}$ can be projected onto the moment space, and the following results can be obtained:

$$
\begin{gathered}
\mathbf{m}=\mathbf{M f}=\left(\rho, e, \varepsilon, j_{x}, q_{x}, j_{y}, q_{y}, p_{x x}, p_{x y}\right)^{T}, \\
\mathbf{m}^{e q}=\mathbf{M f}^{e q}=\left(\rho, e^{e q}, \varepsilon^{e q}, j_{x}, q_{x}^{e q}, j_{y}, q_{y}^{e q}, p_{x x}^{e q}, p_{x y}^{e q}\right)^{T} \\
=\rho\left(1,-2+3|\mathbf{u}|^{2}, 1-3|\mathbf{u}|^{2}, u,-v, u,-v, u^{2}-v^{2}, u v\right)^{T}, \\
5
\end{gathered}
$$


where $\mathbf{f}=\left(f_{1}, f_{2}, \ldots, f_{9}\right)^{T}$ and $\mathbf{f}^{e q}=\left(f_{1}^{e q}, f_{2}^{e q}, \ldots, f_{9}^{e q}\right)^{T} ; \rho$ is the density, $e$ is the energy mode, $\varepsilon$ is related to energy square, $\left(j_{x}, j_{y}\right)$ are the momentum components, $\left(q_{x}, q_{y}\right)$ correspond to energy flux, $\left(p_{x x}, p_{x y}\right)$ are related to the diagonal and off-diagonal components of the stress tensors; $u$ and $v$ are the components of the velocity $\mathbf{u}$, respectively.

For isothermal gas flow, the macroscopic density, momentum, and the shear viscosity are recovered by:

$$
\rho=\sum_{i} f_{i}, \quad \rho \mathbf{u}=\sum_{i} \mathbf{c}_{i} f_{i}, \quad \mu=\rho c_{s}^{2}\left(\tau_{s}-\frac{1}{2}\right) \delta_{t},
$$

where $c_{s}=\sqrt{R T}=c / \sqrt{3}$ is the sound speed, in which $R$ is the gas constant and $T$ is the temperature.

To account for the effect of gas molecule/wall interactions, generally two kinds of corrections on mean free path and/or viscosity are proposed in the literature. Zhang et al.[43] and Guo et al.[44] introduced different effective Knudsen number expressions considering the distances of the gas from the solid walls. Besides, Guo et al.[42] and Li et al.[45] averaged the effect of the Knudsen layer to the whole flowing area and proposed the simplified effective Knudsen number expressions. More discussions on Knudsen layer effect can be found from our previous review paper[15]. In this study, the average effective Knudsen number proposed by Guo et al.[42] is used, and the relaxation time $\tau_{s}$ is chosen as:

$$
\tau_{s}=\frac{1}{2}+\sqrt{\frac{6}{\pi}} N K n \frac{2}{\pi} \arctan \left(\sqrt{2} K n^{-3 / 4}\right),
$$

where $N$ is the grid number in the direction of the characteristic length. Note that the fact that $c=\sqrt{3} c_{s}=\sqrt{3 R T}=\delta_{x} / \delta_{t}=1$ has been used in the above deduction.

\subsection{Boundary conditions}

\subsubsection{Wall boundaries}

The wall boundary conditions are required to appropriately describe the momentum exchanges between gas molecules and solid walls, so as to capture the slip motion and adsorption/desorption of gas molecular in organic shale. In order to take account of the adsorption/desorption as well as the surface diffusion effects that are illustrated in Fig.2, inspired by the work of Levesque et al.[36, 46] and their co-workers[47], we firstly proposed a similar propagation scheme in the slip boundary condition for micro-gas flow.In the new boundary condition, the solid wall is assumed to be covered by a certain amount of adsorbed gas and the adsorption/ desorption is in equilibrium, therefore the density of both free gas and adsorbed gas remains constant. The transport of the adsorbed gas is following the surface diffusion rule and it is assumed to be independent to the transport of the free gas. The influence of premature desorption of adsorbed gas on free gas transport is introduced to the new boundary condition by the definition of a "post-desorption distribution functions" $f_{i}^{\text {des }}(\mathbf{x})$, and $\mathbf{u}_{\text {des }}$ is assumed to be the same as $\mathbf{u}_{\text {surface }}$. The distribution function of fluid boundary nodes $f_{i}^{B C}(\mathbf{x})$ is defined as:

$$
f_{i}^{B C}(\mathbf{x}, \rho, \mathbf{u})=\theta f_{i}^{\text {des }}\left(\mathbf{x}, \rho, \mathbf{u}_{\text {surface }}\right)+(1-\theta) f_{i}^{f r}\left(\mathbf{x}, \rho, \mathbf{u}_{w}\right),
$$

$f_{i}^{f r}(\mathbf{x})$ is the reflected distribution function contributed from from the free gas. $f_{i}^{\text {des }}(\mathbf{x})$ holds the free gas density and with the surface velocity $\mathbf{u}_{\text {surface }}$ according to its definition. 
In order to calculate the distribution function associated with free gas particles $f_{i}^{f r}(\mathbf{x})$, the kinetic boundary condition proposed by Tang et al.[20] is introduced, where $f_{i}^{f r}(\mathbf{x})$ is a combination of the specular reflection distribution function, $f_{i}^{s r}$ with the completely diffusive distribution function, $f_{i}^{d r}$, and it can be expressed as:

$$
f_{i}^{f r}(\mathbf{x})=r f_{i}^{d r}(\mathbf{x})+(1-r) f_{i}^{s r}(\mathbf{x}),\left(\mathbf{c}_{i}-\mathbf{u}_{w}\right) \cdot \mathbf{n}>0,
$$

where the specular reflected distribution function, $f_{i}^{s r}$ satisfies:

$$
f_{i}^{s r}(\mathbf{x})=f_{i^{\prime}}(\mathbf{x}),
$$

with $\mathbf{c}_{i^{\prime}}=\mathbf{c}_{i}-2\left[\left(\mathbf{c}_{i}-\mathbf{u}_{w}\right) \cdot \mathbf{n}\right] \mathbf{n}$ being the specular velocity of $\mathbf{c}_{i}$, and $\mathbf{n}$ is the unit normal vector. The diffusive distribution part is derived by Ansumali and Karlin[48] and can be calculated as:

$$
f_{i}^{d r}(\mathbf{x})=\frac{\sum_{\left(\mathbf{c}_{j}-\mathbf{u}_{w}\right) \cdot \mathbf{n}<0}\left|\left(\mathbf{c}_{j}-\mathbf{u}_{w}\right) \cdot \mathbf{n}\right| f_{j}(\mathbf{x})}{\sum_{\left(\mathbf{c}_{i}-\mathbf{u}_{w}\right) \cdot \mathbf{n}>0}\left|\left(\mathbf{c}_{i}-\mathbf{u}_{w}\right) \cdot \mathbf{n}\right| f_{i}^{e q}(\mathbf{x})} f_{i}^{e q}(\mathbf{x}) .
$$

To match the second order slip boundary condition with a half-way reflection mode, the contribution of the diffusive reflection part, $r$ in Eq. 10 and the relaxation time, $\tau_{q}$ must be chosen as[49]:

$$
\begin{gathered}
r=2\left(1+\sqrt{\frac{\pi}{6}} A_{1}\right)^{-1}, \\
\tau_{q}=\frac{1}{2}+\frac{\pi A_{2}\left(2 \tau_{s}-1\right)^{2}+3}{8\left(2 \tau_{s}-1\right)},
\end{gathered}
$$

where $A_{1}$ and $A_{2}$ are functions of tangential momentum accommodation function, $\sigma$ [44]:

$$
A_{1}=\frac{2-\sigma}{\sigma}(1-0.1817 \sigma)
$$

$$
A_{2}=\frac{2}{\pi}+\frac{1}{2} A_{1}^{2}
$$

For the calculation of "post-desorption distribution functions" $f_{i}^{\text {des }}(\mathbf{x})$, we introduced the boundary condition proposed by Inamuro et al.[50]. $f_{i}^{d e s}(\mathbf{x})$ is assumed to be the equilibrium distribution function with a counter slip velocity to ensure the gas at the fluid boundary has the velocity, $\mathbf{u}_{\text {surface }}$ and the density, $\rho$ :

$$
f_{i}^{\text {des }}(\mathbf{x})=f_{i}^{e q}\left(\mathbf{x}, \rho^{\prime}, u^{\prime}\right),
$$

and for lower fluid boundary nodes, we have:

$$
\begin{gathered}
\rho=\frac{1}{1-v_{\text {surface }}}\left[f_{1}+f_{2}+f_{4}+2\left(f_{5}+f_{8}+f_{9}\right)\right], \\
\rho^{\prime}=6\left[\left(\rho v_{\text {surface }}+f_{5}+f_{8}+f_{9}\right) /\left(1+3 v_{\text {surface }}+3 v_{\text {surface }}^{2}\right)\right], \\
u^{\prime}=\frac{1}{1+3 v_{\text {surface }}}\left\{6\left[\left(\rho u_{\text {surface }}-\left(f_{2}+f_{9}-f_{4}-f_{8}\right)\right) / \rho^{\prime}\right]-u_{\text {surface }}-3 u_{\text {surface }} v_{\text {surface }}\right\},
\end{gathered}
$$


where $u_{\text {surface }}$ and $v_{\text {surface }}$ are the $x$ and $y$ components of the velocity $\mathbf{u}_{\text {surface }}$, respectively. With an assumption that the surface diffusion is along the channel $\left(v_{\text {surface }}=0\right)$, and in consistent with the half-way boundary condition used to calculate $f^{f r}(\mathbf{x}), u_{\text {surface }}$ needs to be replaced with $u_{\text {surface }}^{\prime}$, and $u_{\text {surface }}^{\prime}$ is given as:

$$
u_{\text {surface }}^{\prime}=u_{\text {surface }}+4 u_{\max }\left(\frac{1}{2} \delta x / H\right)\left(1-\left(\frac{1}{2} \delta x / H\right)\right),
$$

where $u_{\max }$ is the maximum streamwise velocity, $H$ is the channel height, and $u_{\text {surface }}$ can be calculated based on Eq. 2.

\subsubsection{Inlet/outlet}

In order to specify the pressure on flow boundaries, the extrapolated boundary conditions[22] are employed at inlet/outlet ends of the channel. The inlet and outlet are located at $x=(1, y)$ and $x=\left(N_{x}, y\right)$ with $y \in\left[1, N_{y}\right]$, the ghost nodes are defined at the positions $x=(0, y)$ and $x=\left(N_{x}+1, y\right)$. The following equations are used to determine the unknown distribution based on a linear extrapolation scheme:

$$
\begin{gathered}
f_{i}(0, y)=2 f_{i}(1, y)-f_{i}(2, y), i=\{2,6,9\}, \\
f_{i}\left(N_{x}+1, y\right)=2 f_{i}\left(N_{x}, y\right)-f_{i}\left(N_{x}-1, y\right), i=\{4,7,8\},
\end{gathered}
$$

after this, in order to realize the pressure boundary condition, the extrapolated density needs to be renormalized to the prescribed density:

$$
\begin{gathered}
\rho_{\text {new }}(1, y)=\frac{N_{y} \rho_{\text {in }}}{\sum_{y=1}^{N_{y}} \rho(1, y)} \rho(1, y), \\
\rho_{\text {new }}\left(N_{x}, y\right)=\frac{N_{y} \rho_{\text {out }}}{\sum_{y=1}^{N_{y}} \rho(1, y)} \rho\left(N_{x}, y\right) .
\end{gathered}
$$

The renormalized values are used in the equilibria to carry out the ensuring collision step. This procedure ensures the pressure profile is consistent with flow field inside the channel[22].

\section{Numerical validation}

The simulation runs are carried out by assuming $\theta=0$ and the results are compared with that of DSMC and IP-DSMC results as reported by Shen et al. [51], which are suitable for modelling micro-gaseous flow and have been extensively used for such validation purposes. In this case, the ratio of the length to the height, $L / H$ is 100 , and a uniform lattice $N_{x} \times N_{y}=2100 \times 21$ is adopted. The outlet gas density $\rho_{\text {out }}$ is 1 , and pressure ratio of inlet and outlet $p_{\text {in }} / p_{\text {out }}$ is taken as 1.4 for $K n_{\text {out }}=0.0194$ and 2 for $K n_{\text {out }}=0.194$, respectively. The convergence criterion towards the steady state is set as follows:

$$
\sqrt{\frac{\sum_{i}\left|\mathbf{u}\left(\mathbf{x}_{i}, t_{n}\right)-\mathbf{u}\left(\mathbf{x}_{i}, t_{n}-1000 \delta t\right)\right|^{2}}{\sum_{i}\left|\mathbf{u}\left(\mathbf{x}_{i}, t_{n}\right)\right|^{2}}}<10^{-12}
$$


The normalized streamwise velocity $U=u / u_{\max }$ predicted by this study for $K n_{\text {out }}=0.0194$ and $K n_{\text {out }}=0.194$ are shown in Fig. 3. It can be seen from the figure that the overall velocity profile are in good agreement with those of the DSMC/IP-DSMC and the LBM is applicable for slip flow and the early part of transition regime which covers a large portion of flow in shale.

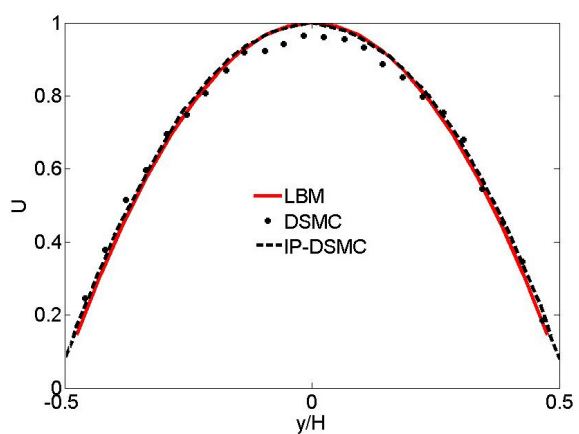

(a) $K n_{\text {out }}=0.0194$

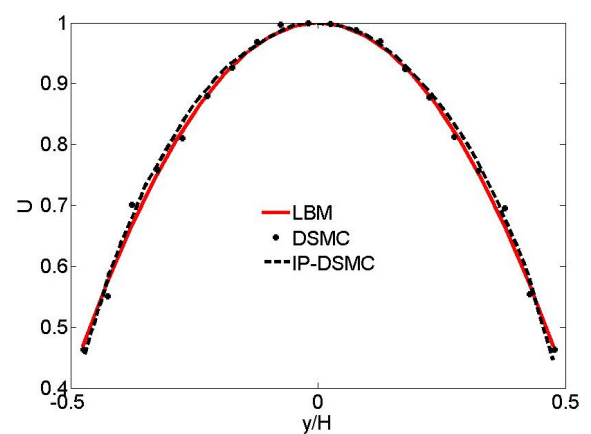

(b) $K n_{\text {out }}=0.194$

Figure 3: Normalized streamwise velocity at different Knudsen number

The molar fluxes through a channel with $H=5 \mathrm{~nm}$ and $L=50 \mathrm{~nm}$ at $T=338.7 \mathrm{k}$ predicted by DGM under different pressures are also presented for validation. By the superposition of convective transport and Knudsen diffusion, Veltzke and Thöming[52] proposed the DGM for channel and tube flow and concluded that the mass flux estimated by DGM fits the experimental data well in the slip regime and the beginning of transition regime. The molar flux estimated based on DGM for a single channel can be written as:

$$
N_{D G M}=-\frac{1}{R T}\left(D_{k}+\frac{K_{0}}{\eta} P\right) \nabla P,
$$

where $D_{k}$ is Knudsen diffusivity, $K_{0}$ is the absolute permeability, and $\eta$ is viscosity. The comparisons are shown in Fig.4, and the simulation results estimated based on LB model are comparable to those of the DGM. 


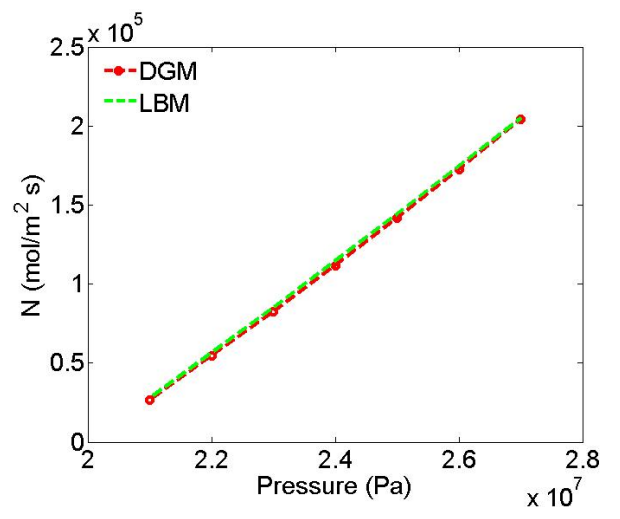

(a) $P_{\text {out }}=20 \mathrm{MPa}$

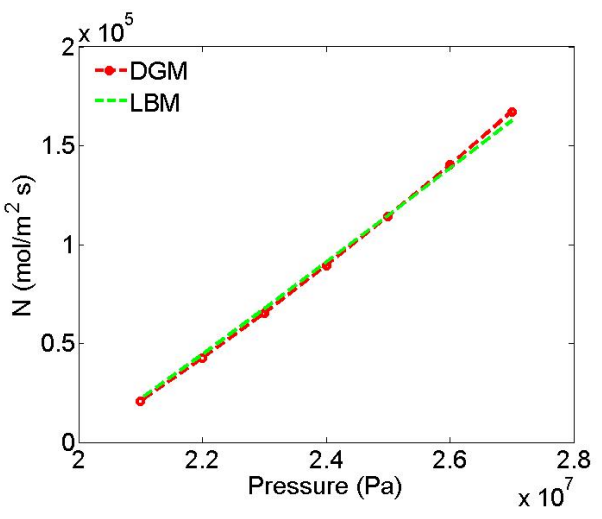

(b) $P_{\text {out }}=10 \mathrm{MPa}$

Figure 4: Molar flux at different pressure (T=338.7k, $\mathrm{H}=5 \mathrm{~nm}, \mathrm{~L}=50 \mathrm{~nm}$.)

\section{Simulation examples}

\subsection{Influence of adsorbed gas and surface diffusion on micro-gaseous flow}

In order to qualify the influence of adsorbed gas and its surface diffusion on micro-gaseous flow, in this section, the normalized streamwise velocity distribution of the free gas and the total molar flux at different $\theta$ and $D_{s}$ are analysed. Methane flow through channels with $P_{\text {in }}=22 \mathrm{MPa}$ and $P_{\text {out }}=20 \mathrm{MPa}$ at $T=338.7 \mathrm{~K}$ are considered. $N_{x} \times N_{y}=41 \times 803$ lattice and $N_{x} \times N_{y}=$ $41 \times 403$ lattice are adopted to discretize $5 \times 100 \mathrm{~nm}$ and $10 \times 100 \mathrm{~nm}$ micro-channels, respectively. Extrapolated boundary conditions are applied to the inlet and outlet boundaries, and the proposed boundary condition is applied to the solid wall. Before starting the simulation, $f^{e q}$ with $\mathbf{u}=0$ and $\rho$ satisfying linear distribution from $\rho_{\text {in }}$ and $\rho_{\text {out }}$ are adopted as the initial value of distribution function $f_{i}$ at each lattice. Based on the fact that the adsorbed gas molecules need to overcome additional resistance of the wall, the surface diffusion velocity is assumed to be no larger than the slip velocity without the consideration of adsorption and surface diffusion[10, 25], and the normalized slip velocity satisfies $U_{s}=4 A_{1} K_{n}+8 A_{2} K_{n}$ [49]. When the gas adsorption reaches a maximum, the density of the adsorbed gas $\rho_{a d s, s a t}$ is assumed to be $340 \mathrm{~kg} / \mathrm{m}^{3}$ according to Ambrose et al.'s work[53], and therefore the density of the adsorbed gas at different $\theta$ satisfies $\rho_{a d s}=\theta \rho_{a d s, s a t}$.

\subsubsection{Influence of $\theta$ and $D_{s}$ on normalised streamwise velocity}

The normalised stream-wise velocities of the free gas at different adsorbed gas coverages and different surface diffusion velocities are shown in Fig.5. When $\theta=0$, the variation of $u_{\text {surface }}$ does not influence the free gas flow because there is no adsorbed gas in the system (Fig.5 (a)). The gas slip at the solid surface is controlled by the interaction of free gas molecules with solid molecules and the rarefaction dominate the flow which leads to a large slip velocity at the boundary. When $\theta=1$, the slip velocity of the free gas at the solid surface is completely determined by the desorbed gas, and it vanishes to zero at $u_{\text {surface }}=0$ (Fig.5 $(d)$ ). This results is consistent with a very recent MD study performed by Wang et al.[11], who claimed that the gas flow in confined micro-channel manifests a parabolic velocity profile with zero slip velocity at the solid surface when an immobile adsorption layer is formed, and the slip model based on kinetic theory[54] 
fails to capture the flow behavior. When the solid surface is partially covered by an amount of adsorbed gas, the slippage of the free gas is determined by the interactions with both solid wall and the adsorbed gas, and the "dynamic similarity" of rarefied gas flow and micro-scale confined flow breaks down. At a certain coverage, the gas slip at the wall decreases with the decreased $u_{\text {surface }}$ as a result of the premature short-time desorption of adsorbed gas (Fig.5 $(b)$ and $(c)$ ). Compared different sub-figures in Fig.5, when $u_{\text {surface }}=u_{s}$, the increase with coverage does not influence the slip velocity as according to the assumption of $\mathbf{u}_{\text {des }}=\mathbf{u}_{\text {surface }}$, otherwise, the slip of the free gas is decreased with the increase of adsorbed gas coverage $\theta$ at a certain $u_{\text {surface }}$. Previous MD studies have concluded that the near wall velocity profile shows reduced gas slip with the increased surface-gas potential strength/ more explicit adsorption[1, 11, 13, 55, 56], and this phenomenon is confirmed by current LB simulation results in this study.

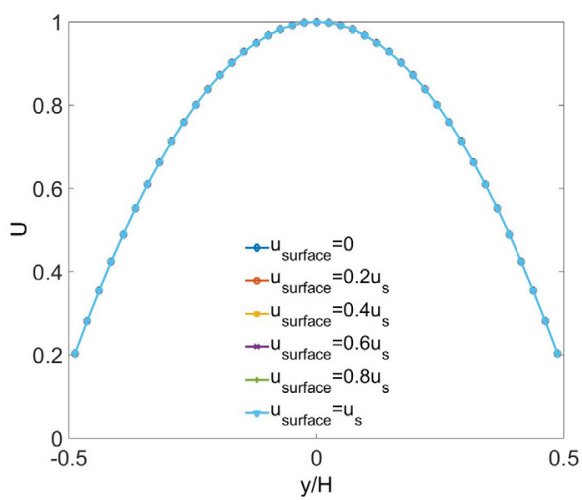

(a) Coverage, $\theta=0$

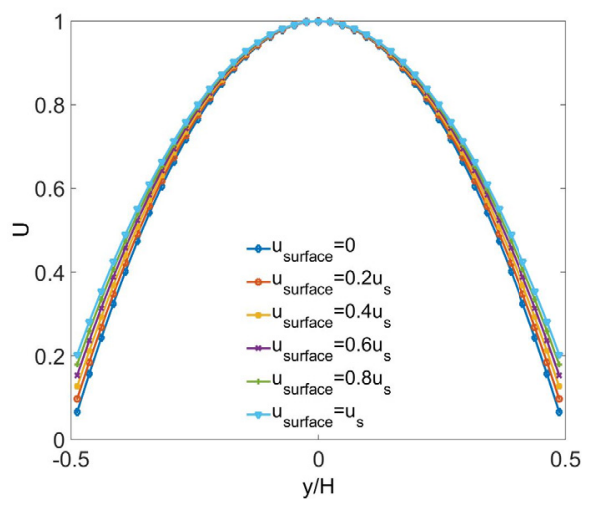

(c) Coverage, $\theta=0.6$

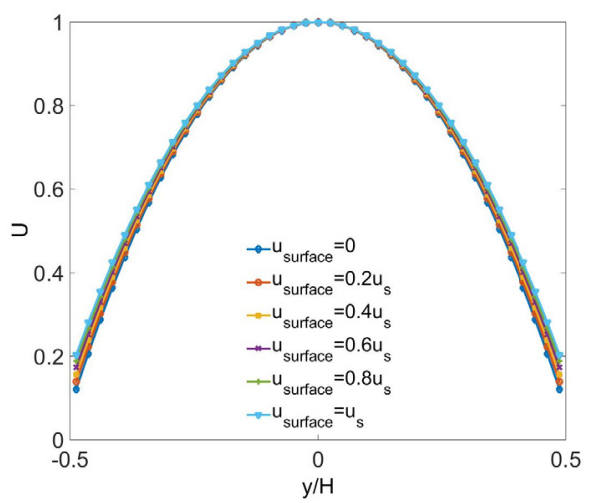

(b) Coverage, $\theta=0.2$

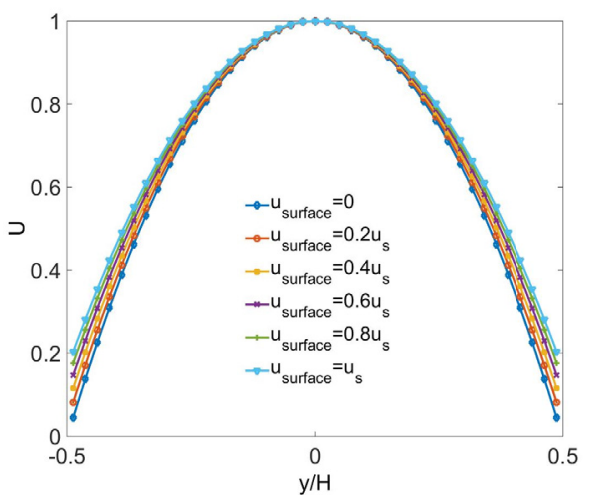

(d) Coverage, $\theta=1$

Figure 5: Normalized velocity $\mathrm{U}$ at different surface coverage $\theta$ and surface diffusion velocity $u_{\text {surface }}$. Width=5nm, length $=100 \mathrm{~nm}, \mathrm{~T}=338.7 \mathrm{~K}, P_{\text {in }}=22 \mathrm{MPa}$ and $P_{\text {out }}=20 \mathrm{MPa}$.

\subsubsection{Influence of $\theta$ and $D_{s}$ on molar flux}

The molar flux of the free gas $N_{\text {free }}$, the molar flux of the adsorbed gas $N_{\text {surface }}$ and the total molar flux $N_{\text {free }}+N_{\text {surface }}$ at different $\theta$ and $u_{\text {surface }}$ of $5 \mathrm{~nm}$ and $10 \mathrm{~nm}$ wide channels are shown 
in Figs. 6 and 7, respectively. In Fig. $6(a)$ and Fig. $7(a)$, the variations of $N_{\text {free }}$ with $\theta$ are consistent with the velocity profiles shown in Fig.5. It remains as a constant at different $\theta$ when $u_{\text {surface }}=u_{s}$, and it decreases with the increase of $\theta$ because the $u_{\text {surface }}$ is assumed to be smaller than $u_{s}$. $N_{\text {surface }}=0$ at $u_{\text {surface }}=0$ as the adsorbed gas is immobile, otherwise, $N_{\text {surface }}$ shows a linearly increase with the increase of $\theta$ because the adsorbed gas density close to the solid surface is much larger at a larger $\theta$. Also, $N_{\text {surface }}$ increases with the increase of $u_{\text {surface }}$ as a larger $u_{\text {surface }}$ indicates a stronger mobility of the adsorbed gas. Compared $N_{\text {free }}$ with $N_{\text {surface }}$, it can be concluded that the molar flux produced by surface diffusion is more significant than that of free gas at a large adsorption coverage if the adsorbed gas is mobile in the system. In a $5 \mathrm{~nm}$ wide channel, the $N_{\text {surface }}$ can exceed $N_{\text {free }}$ at $u_{\text {surface }}=u_{s}$ and $u_{\text {surfaec }}=0.8 u_{s}$ if the solid surface is covered by enough adsorbed gas (Fig.6(a)). In Fig. $6(b)$ and Fig. $7(b)$, the variation of $N_{\text {total }}$ shows a changeable trend with $\theta$ at different $u_{\text {surface }}$. At low $u_{\text {surface }}, N_{\text {total }}$ decreases with $\theta$ because the adsorbed gas contributes very little to the total mass flow. However, at high $u_{\text {surface }}$, since the adsorbed gas density is much larger than that of free gas, the adsorbed gas contributes substantial mass flux, and $N_{\text {total }}$ starts to increase with $\theta$. These results are consistent with a recent non-equilibrium molecular dynamics (NEMD) study on gas transport in nanopores. Wu and Zhang[12] performed several NEMD simulations on gas flow through illite and graphene channels, and they concluded that the total mass flux is significantly influenced by the gas mobility in adsorption region, and the mass flux can either be enhanced or reduced by adsorbed gas.

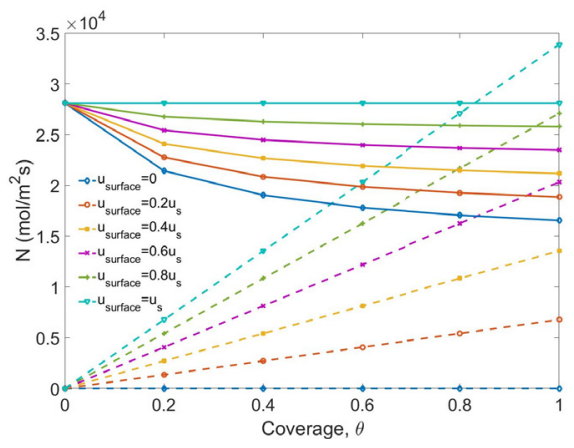

(a) $N_{\text {surface }}\left(\right.$ dash line) and $N_{\text {free }}$ (solid line)

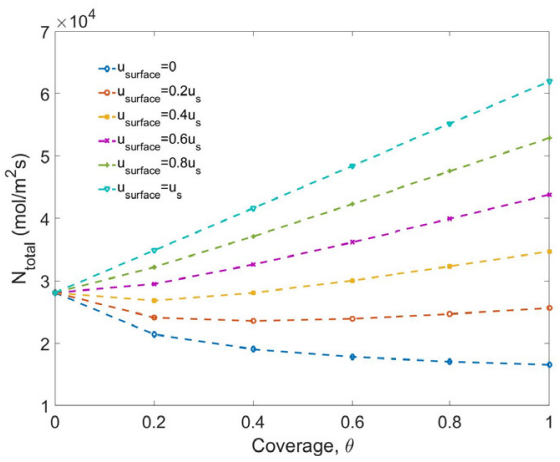

(b) $N_{\text {total }}$

Figure 6: Variation of molar flux with adsorption gas coverage at different surface diffusion velocity. The molar flux of free gas is indicated by solid line and the molar flux of adsorbed gas is indicated by dash line. Width $=5 \mathrm{~nm}$, length $=100 \mathrm{~nm}$, $T=338.7 \mathrm{~K}, P_{\text {in }}=22 \mathrm{MPa}$ and $P_{\text {out }}=20 \mathrm{MPa}$. 


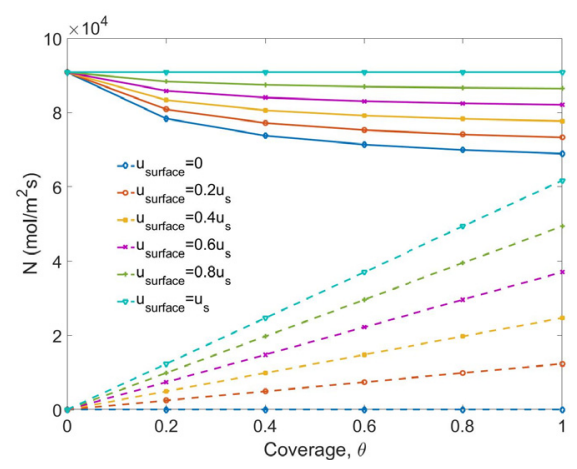

(a) $N_{\text {surface }}$ (dash line) and $N_{\text {free }}$ (solid line)

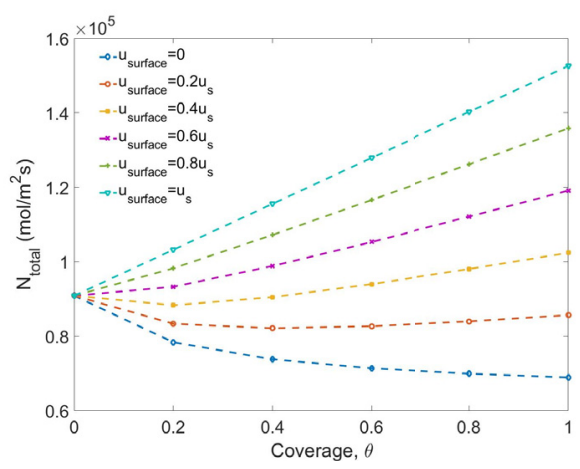

(b) $N_{\text {total }}$

Figure 7: Variation of molar flux with adsorption gas coverage at different surface diffusion velocity. The molar flux of free gas is indicated by solid line and the molar flux of adsorbed gas is indicated by dash line. Width=10nm, length $=100 \mathrm{~nm}, \mathrm{~T}=338.7 \mathrm{~K}, P_{\text {in }}=22 \mathrm{MPa}$ and $P_{\text {out }}=20 \mathrm{MPa}$.

\subsection{Application of the LBM to study gas flow in organic shale}

In this section, the LB model with the proposed boundary condition is used to study gas flow in organic shale considering the surface diffusion of adsorbed gas. It is noted that the gas flow in porous shale is conventionally modelled as capillaries. Here, pressure driven methane flow through $100 \mathrm{~nm}$ long micro-channels with width ranges from $5 \mathrm{~nm}$ to $40 \mathrm{~nm}$ are considered, and extrapolated boundary conditions are applied to the inlet and outlet boundaries. The outlet pressure ranges from $10 \mathrm{MPa}$ to $50 \mathrm{MPa}$, and the inlet pressure is $2 \mathrm{MPa}$ higher than outlet pressure. Langmuir adsorption isotherm model is used to describe the adsorption of methane into shale matrix, and the Maxwell-Stefan approach is applied to describe the transport of adsorbed gas. Therefore, $\theta=b P /(1+b P)$ and $u_{\text {surface }}$ can be expressed as a function of Langmuir properties and surface diffusivity with Eq. 2. Because the apparent permeability of gas is one of the most important petro-physical parameters to porous shale, and its variation with rock properties and production conditions usually attracts many discussions, the influences of pore width, pressure and Langmuir properties on gas apparent permeability are discussed. Based on a sensitivity analysis, parameters affecting apparent permeability of organic shale are quantified. The apparent permeability is calculated with $k_{\text {app }}=N_{\text {total }} M \mu /\left(\rho_{\text {free }} \nabla P\right)$. Since the specific values of surface diffusivity and Langmuir property of shale samples are still not clear and more experiments will likely be done in the future, in this study a wide range of parameter analysis is considered. $D_{s}$ from 0 to $1 \times 10^{-5} \mathrm{~m}^{2} / \mathrm{s}, b$ from 0.125 to $1 \mathrm{MPa}^{-1}$ and $q_{\text {sat }}$ from 1000 to $8000 \mathrm{~mol} / \mathrm{m}^{3}$ [23] are used for the following discussions.

\subsubsection{Influence of the pore width}

In order to study the influence of pore width on gas apparent permeability, the variations of $K_{a p p}$ and the ratio of $K_{a p p}$ to intrinsic permeability $K_{0}$ with pore width at different surface diffusivities are simulated using the LB model developed in this study and the results are shown in Figs.8 and 9. The permeability without adsorbed gas is also presented for the purpose of comparison. Temperature, the Langmuir volume $\left(q_{\text {sat }}\right)$, and the Langmuir constant $(b)$ are set to be $338.7 \mathrm{~K}, 8000 \mathrm{~mol} / \mathrm{m}^{3}$ and $1 \times 10^{-6} \mathrm{~Pa}^{-1}$, respectively. From Figs. 8 and 9 it can be seen that, at low surface diffusivity, pore width has a negligible effect on $K_{a p p}$. When surface diffusion is noticeable, the pore width has a significant influence on both $K_{a p p}$ and $K_{0}$. With the increase 
in pore width, both $K_{a p p}$ and $K_{0}$ increase, and the $K_{a p p} / K_{0}$ reaches a Plateau at pore diameter of about $40 \mathrm{~nm}$. This behaviour can be explained by the fact that in larger pores, the flux from viscous flow is predominant and the surface effects which include the gas slip and the surface diffusion are almost negligible. As a consequent of this, $K_{a p p}$ becomes approximately equal to $K_{0}$. Moreover, the effect of pore diameter on $K_{a p p}$ is more pronounced at high surface diffussivity especially at low pressures, and difference in $K_{a p p}$ and $K_{0}$ is increased with the increase of $D_{s}$ in a certain pore space. Comparing cases with and without adsorbed gas, $K_{a p p}$ considering adsorbed gas is smaller than that without considering adsorbed gas at low $D_{s}$. When $D_{s}$ reaches an order of order of $10^{-6} \mathrm{~m}^{2} / \mathrm{s}$, with the increase of $D_{s}$, a significant underestimation of $K_{a p p}$ can be observed at high surface diffusivity in small pores, which is in good agreement with previous pore-scale numerical studies $[8,57]$.

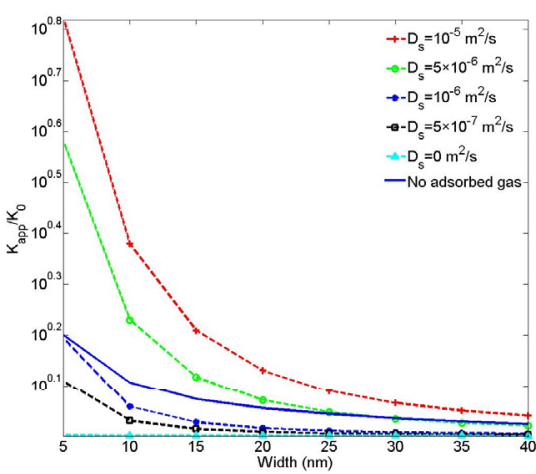

(a) $K_{\text {app }} / K_{0}$

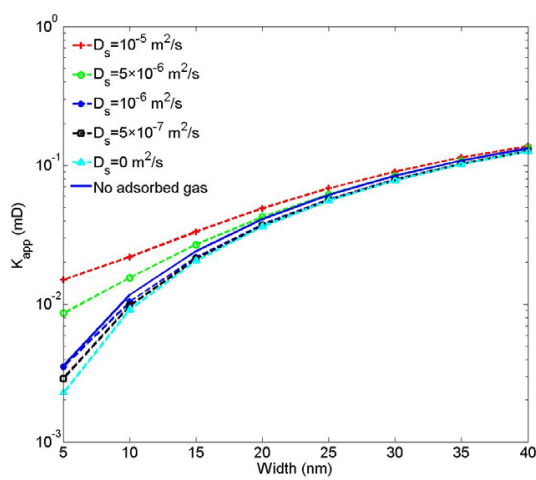

(b) $K_{a p p}$

Figure 8: $K_{\text {app }} / K_{0}$ and $K_{\text {app }}$ at $P_{\text {in }}=22 \mathrm{MPa}$ and $P_{\text {out }}=20 \mathrm{MPa}$

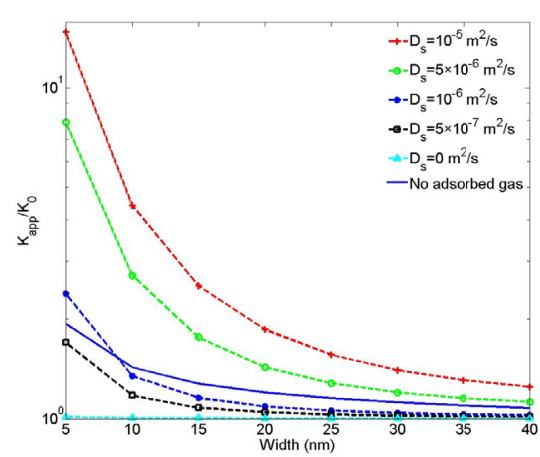

(a) $K_{\text {app }} / K_{0}$

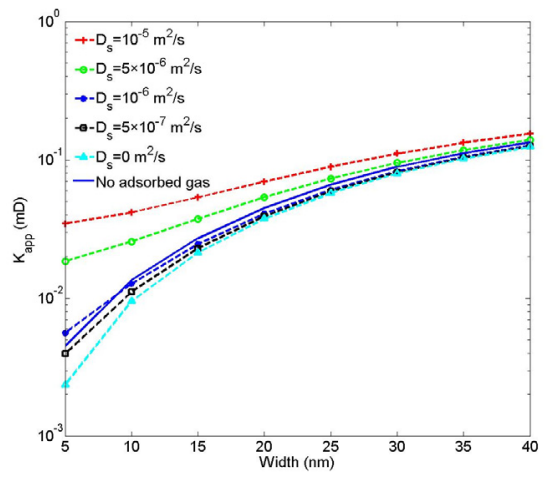

(b) $K_{a p p}$

Figure 9: $K_{a p p} / K_{0}$ and $K_{\text {app }}$ at $P_{\text {in }}=12 \mathrm{MPa}$ and $P_{\text {out }}=10 \mathrm{MPa}$ 


\subsubsection{Influence of pressure}

The variations of $K_{a p p}$ and $K_{a p p} / K_{0}$ with average pressure are shown in Figs. 10 and 11. Temperature, Langmuir properties and surface diffusivity are the same as those in Sec.5.2.1. Figs. 10 and 11 illustrate that, at extreamly low surface diffusivity, as the immobile adsorbed gas restricts the surface flow, the effect of pressure on the $K_{a p p}$ is deminished and $K_{a p p}$ approximately equals $K_{0}$. Otherwise, since the increased pressure reduces the mobility of adsorbed gas and the rarefication, the $K_{a p p}$ and $K_{a p p} / K_{0}$ decrease with the increase in average pressure especially in smaller pores, which is consistent with the macroscopic behavior of shale gas production[58]. At low pressure, both gas slip and the surface diffusion of adsorbed gas contribute to the increased $K_{a p p}$, and when $D_{s}$ reaches to an order of $10^{-6} \mathrm{~m}^{2} / \mathrm{s}$, the $K_{a p p}$ is larger than cases without adsorbed gas. The results also indicate that, at high pressure conditions such as reservoir condition, although the rarefication and slip effects are negligible, the surface diffusion of adsorbed gas can have significant contribution of total mass flux and finally leads to a large $K_{a p p}$ compared to $K_{0}$ at high surface diffusivity.

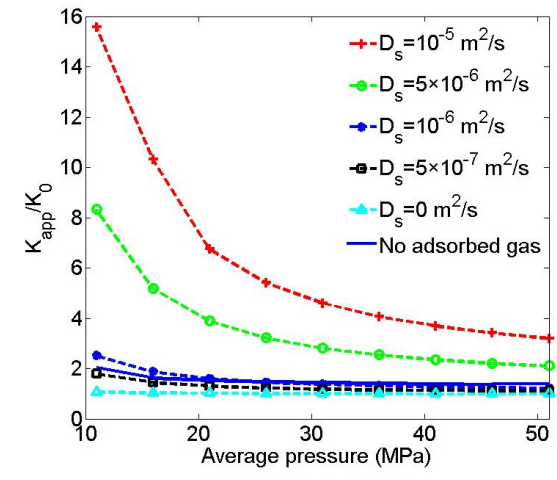

(a) $K_{\text {app }} / K_{0}$

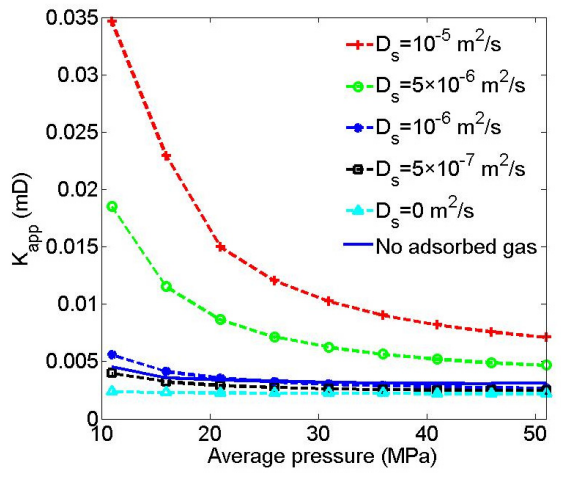

(b) $K_{\text {app }}$

Figure 10: $K_{a p p} / K_{0}$ and $K_{a p p}$ at Width $=5 \mathrm{~nm}$ and Length $=100 \mathrm{~nm}$

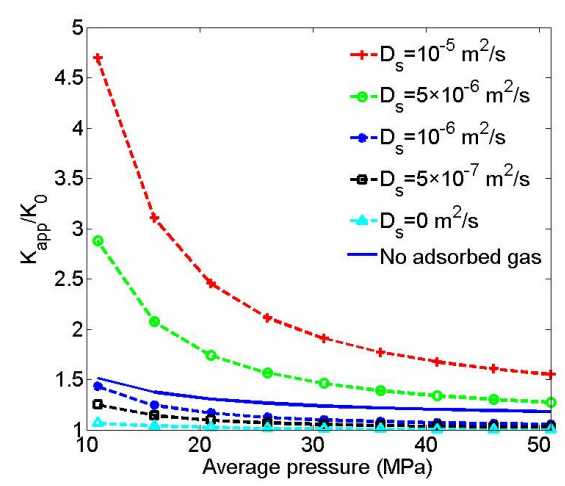

(a) $K_{\text {app }} / K_{0}$

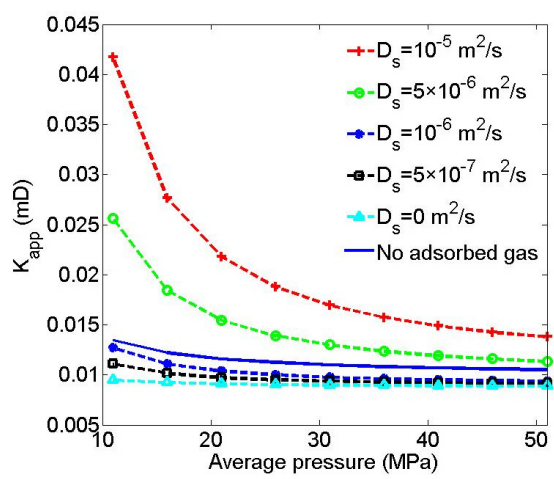

(b) $K_{a p p}$

Figure 11: $K_{\text {app }} / K_{0}$ and $K_{\text {app }}$ at Width $=10 \mathrm{~nm}$ and Length $=100 \mathrm{~nm}$ 


\subsubsection{Influence of Langmuir properties}

In an effort to better understand the role of adsorbed gas on apparent permeability of shale, the effects of Langmuir volume $\left(q_{s a t}\right)$ and Langmuir constant $(b)$ on $K_{a p p}$ are evaluated and the results are shown in Figs.12 and 13. The inlet pressure, outlet pressure, the pore width, and length are set as $22 \mathrm{MPa}, 20 \mathrm{MPa}, 10 \mathrm{~nm}$ and $100 \mathrm{~nm}$, respectively. From Fig.12 it can be seen that for constant value of $b$, an increase in $q_{\text {sat }}$ leads to an increase in $u_{\text {surface }}$ which results in a larger $K_{a p p}$, especially at high surface diffusivities. This is also evident in Eq.2 that $q_{\text {sat }}$ is the controlling parameter for velocity of adsorbed gas. The influence of $b$, however, is of two folds: $b$ controls both $\theta$ and $u_{\text {surface }}$ at a certain pressure condition. Fig. 13 shows that, with low surface diffusivity, the influence of $b$ on $u_{\text {surface }}$ is negligible, and that the $K_{a p p}$ decreases with $b$ as more gas tends to be adsorbed on the solid surface. As $D_{s}$ increases, the effect of $b$ on $u_{\text {surface }}$ becomes noticeable, and the $K_{a p p}$ starts to increase with $b$.

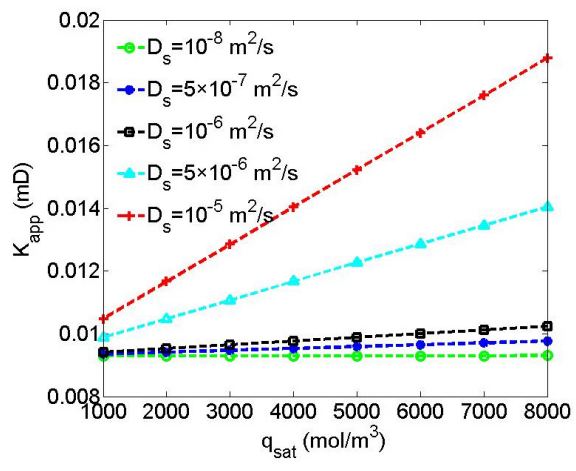

(a) $b=0.125 \times 10^{-6} \mathrm{~Pa}^{-1}$

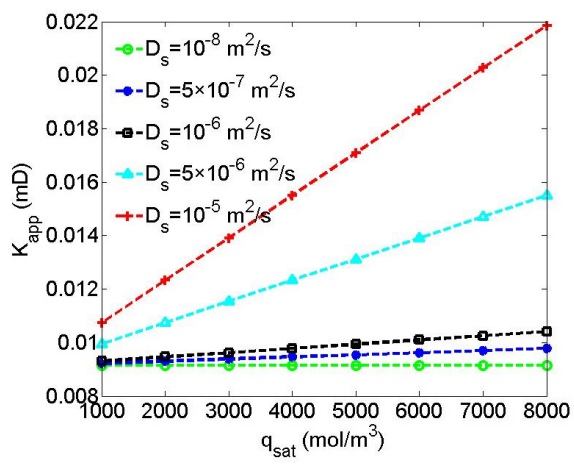

(b) $b=10^{-6} \mathrm{~Pa}^{-1}$

Figure 12: Variation of $K_{a p p}$ with $q_{\text {sat }}$

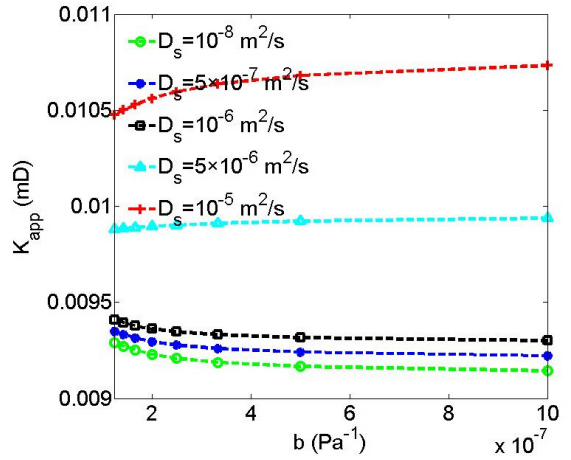

(a) $q_{\text {sat }}=1000 \mathrm{~mol} / \mathrm{m}^{3}$

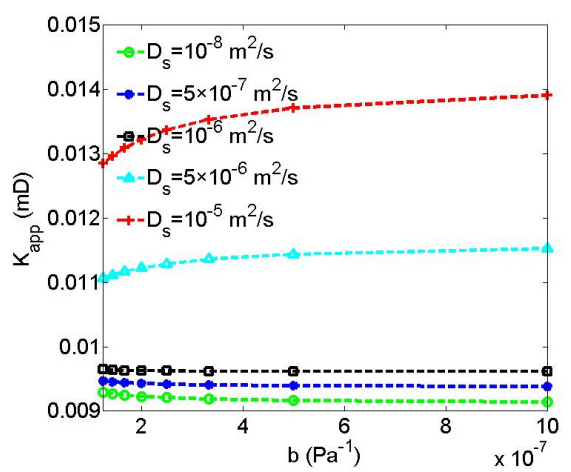

(b) $q_{\text {sat }}=8000 \mathrm{~mol} / \mathrm{m}^{3}$

Figure 13: Variation of $K_{a p p}$ with $b$ 


\section{Conclusion}

In this study, a MRT lattice Boltzmann model with a new boundary condition to account for adsorbed gas and surface diffusion effects is employed to study gas flow in organic shale and to estimate apparent permeability. The effects of pore width, pressure, and Langmuir properties on apparent permeability have been carried out.

By introducing an extra "post-desorption term" in kinetic boundary condition to reflect adsorbed gas with the aid of Langmuir adsorption model, the results of current LB model match reasonably well with previous studies and capture critical phenomena observed by MD simulations. Neglecting the adsorbed gas results in slip of the free gas, and it is consistent with the kinetic theory predictions. Presence of adsorbed gas breaks down the dynamic similarity between rarefied and nano-/micro-scale gas flows based on the Knudsen numbers. Current LB model shows reduced gas slip with the decrease of surface diffusion velocity and stick when an immobile adsorbed gas layer is formed near the solid surface. The new boundary condition overcomes the shortage of previous slip boundary conditions in LB model that indicates substantial velocity slip on the solid walls.

The sensitivity studies confirm that, unlike the intrinsic permeability, the apparent permeability of organic shale is a dynamic parameter and should be adjusted during the production. The apparent permeability increases with the increase of pore radius and the decrease of pressure, and it is controlled by the Langmuir properties. The adsorbed gas and its surface diffusion have a profound effect in determining the apparent permeability, and the gas apparent permeability can be either enhanced or reduced depending on variations in surface diffusion of adsorbed gas under different conditions.

Results of this study can help improve our understanding on solid-gas interactions near the wall and gas adsorption as well as surface diffusion in organic shale. The proposed LB model will be extended in porous shale in future studies.

\section{Acknowledgements}

The authors thank the support from SCOPE, UNSW and the LDRD program of LANL. J.W. thanks the financial support from the China Scholarship Council (CSC). L.C. thanks the support from National Nature Science Foundation of China (No. 51406145, 51136004), and Q.K. thanks the support from a DOE oil \& gas project.

\section{References}

[1] M. Barisik, A. Beskok, Surface-gas interaction effects on nanoscale gas flows, Microfluidics and nanofluidics 13 (5) (2012) 789-798.

[2] F. Javadpour, D. Fisher, M. Unsworth, Nanoscale gas flow in shale gas sediments, Journal of Canadian Petroleum Technology 46 (10) (2007) 55-61.

[3] A. Beskok, G. E. Karniadakis, Report: a model for flows in channels, pipes, and ducts at micro and nano scales, Microscale Thermophysical Engineering 3 (1) (1999) 43-77.

[4] F. Civan, C. S. Rai, C. H. Sondergeld, Shale gas permeability and diffusivity inferred by improved formulation of relevant retention and transport mechanisms, Transport in Porous Media 86 (3) (2011) 925-944.

[5] P. Charoensuppanimit, S. A. Mohammad, K. A. Gasem, Measurements and modeling of gas adsorption on shales, Energy \& Fuels 30 (3) (2016) 2309-2319.

[6] Y. Wu, J. Li, D. Ding, C. Wang, Y. Di, A generalised framework model for the simulation of gas production in unconventional gas reservoirs, SPE Journal 19 (05) (2014) 845-857.

[7] Z. Zhai, X. Wang, X. Jin, L. Sun, J. Li, D. Cao, Adsorption and diffusion of shale gas reservoirs in modeled clay minerals at different geological depths, Energy \& Fuels 28 (12) (2014) 7467-7473. 
[8] K. Wu, X. Li, C. Wang, W. Yu, Z. Chen, Model for surface diffusion of adsorbed gas in nanopores of shale gas reservoirs, Industrial \& Engineering Chemistry Research 54 (12) (2015) 3225-3236.

[9] R. Krishna, J. Wesselingh, The maxwell-stefan approach to mass transfer, Chemical Engineering Science 52 (6) (1997) 861-911.

[10] J. Ren, P. Guo, Z. Guo, Z. Wang, A lattice boltzmann model for simulating gas flow in kerogen pores, Transport in Porous Media (2014) 1-17.

[11] S. Wang, Q. Feng, F. Javadpour, Y.-B. Yang, Breakdown of fast mass transport of methane through calcite nanopores, The Journal of Physical Chemistry C 120 (26) (2016) 14260-14269.

[12] T. Wu, D. Zhang, Impact of adsorption on gas transport in nanopores, Scientific Reports 6.

[13] M. Barisik, A. Beskok, law of the nano-wall in nano-channel gas flows, Microfluidics and Nanofluidics 20 (3) (2016) 1-9.

[14] J. Zhang, Lattice boltzmann method for microfluidics: models and applications, Microfluidics and Nanofluidics 10 (1) (2011) 1-28.

[15] J. Wang, L. Chen, Q. Kang, S. S. Rahman, The lattice boltzmann method for isothermal micro-gaseous flow and its application in shale gas flow: a review, International Journal of Heat and Mass Transfer 95 (2016) 94-108.

[16] L. Chen, W. Fang, Q. Kang, J. D. Hyman, H. S. Viswanathan, W.-Q. Tao, Generalized lattice boltzmann model for flow through tight porous media with klinkenberg's effect, Physical Review E 91 (3) (2015) 033004.

[17] L. Chen, Q. Kang, Z. Dai, H. S. Viswanathan, W. Tao, Permeability prediction of shale matrix reconstructed using the elementary building block model, Fuel 160 (2015) 346-356.

[18] L. Chen, Q. Kang, R. Pawar, Y.-L. He, W.-Q. Tao, Pore-scale prediction of transport properties in reconstructed nanostructures of organic matter in shales, Fuel 158 (2015) 650-658.

[19] L. Chen, L. Zhang, Q. Kang, H. S. Viswanathan, J. Yao, W. Tao, Nanoscale simulation of shale transport properties using the lattice boltzmann method: permeability and diffusivity, Scientific reports 5 .

[20] G. Tang, W. Tao, Y. He, Lattice boltzmann method for gaseous microflows using kinetic theory boundary conditions, Physics of Fluids (1994-present) 17 (5) (2005) 058101.

[21] S. Succi, Mesoscopic modeling of slip motion at fluid-solid interfaces with heterogeneous catalysis, Physical review letters 89 (6) (2002) 064502.

[22] F. Verhaeghe, L.-S. Luo, B. Blanpain, Lattice boltzmann modeling of microchannel flow in slip flow regime, Journal of Computational Physics 228 (1) (2009) 147-157.

[23] J. Wang, L. Chen, Q. Kang, S. S. Rahman, Apparent permeability prediction of organic shale with generalized lattice boltzmann model considering surface diffusion effect, Fuel 181 (2016) 478-490.

[24] A. M. Allan, G. Mavko, The effect of adsorption and knudsen diffusion on the steady-state permeability of microporous rocks, Geophysics 78 (2) (2013) D75-D83.

[25] Z.-Z. Li, T. Min, Q. Kang, Y.-L. He, W.-Q. Tao, Investigation of methane adsorption and its effect on gas transport in shale matrix through microscale and mesoscale simulations, International Journal of Heat and Mass Transfer 98 (2016) 675-686.

[26] E. Fathi, Lattice boltzmann method for simulation of shale gas transport in kerogen, SPE Journal 18 (1) (2013) 27-37.

[27] S. Chen, Z. Tian, Simulation of microchannel flow using the lattice boltzmann method, Physica A: Statistical Mechanics and its Applications 388 (23) (2009) 4803-4810.

[28] S. Brull, P. Charrier, L. Mieussens, Gas-surface interaction and boundary conditions for the boltzmann equation, Kinetic and related models 7 (2) (2014) 422-450.

[29] J. C. Maxwell, On stresses in rarified gases arising from inequalities of temperature, Philosophical Transactions of the royal society of London 170 (1879) 231-256.

[30] W.-M. Zhang, G. Meng, X. Wei, A review on slip models for gas microflows, Microfluidics and nanofluidics 13 (6) (2012) 845-882.

[31] S. K. Bhatia, M. R. Bonilla, D. Nicholson, Molecular transport in nanopores: a theoretical perspective, Physical Chemistry Chemical Physics 13 (34) (2011) 15350-15383.

[32] B.-Y. Cao, Non-maxwell slippage induced by surface roughness for microscale gas flow: a molecular dynamics simulation, Molecular Physics 105 (10) (2007) 1403-1410.

[33] B.-Y. Cao, J. Sun, M. Chen, Z.-Y. Guo, Molecular momentum transport at fluid-solid interfaces in mems/nems: a review, International journal of molecular sciences 10 (11) (2009) 4638-4706.

[34] V. Sokhan, D. Nicholson, N. Quirke, Fluid flow in nanopores: an examination of hydrodynamic boundary conditions, The Journal of Chemical Physics 115 (8) (2001) 3878-3887.

[35] K. Higashi, H. Ito, J. Oishi, Surface diffusion phenomena in gaseous diffusion. i. surface diffusion of pure gas, Nippon Genshiryoku Gakkaishi (Japan) 5.

[36] M. Levesque, M. Duvail, I. Pagonabarraga, D. Frenkel, B. Rotenberg, Accounting for adsorption and desorption in lattice boltzmann simulations, Physical Review E 88 (1) (2013) 013308

[37] I. Langmuir, The adsorption of gases on plane surfaces of glass, mica and platinum., Journal of the American 
Chemical society 40 (9) (1918) 1361-1403.

[38] S. Brunauer, P. H. Emmett, E. Teller, Adsorption of gases in multimolecular layers, Journal of the American chemical society 60 (2) (1938) 309-319.

[39] D. Theodorou, J. Wei, Diffusion and reaction in blocked and high occupancy zeolite catalysts, Journal of Catalysis 83 (1) (1983) 205-224.

[40] J.-H. Moon, Y.-J. Park, M.-B. Kim, S.-H. Hyun, C.-H. Lee, Permeation and separation of a carbon dioxide/nitrogen mixture in a methyltriethoxysilane templating silica/ $\alpha$-alumina composite membrane, Journal of membrane science 250 (1) (2005) 195-205.

[41] A. Marković, D. Stoltenberg, D. Enke, E.-U. Schlünder, A. Seidel-Morgenstern, Gas permeation through porous glass membranes, Journal of Membrane Science 1 (336) (2009) 32-41.

[42] Z. Guo, T. Zhao, Y. Shi, Physical symmetry, spatial accuracy, and relaxation time of the lattice boltzmann equation for microgas flows, Journal of Applied physics 99 (7) (2006) 074903.

[43] Y.-H. Zhang, X.-J. Gu, R. W. Barber, D. R. Emerson, Capturing knudsen layer phenomena using a lattice boltzmann model, Physical Review E 74 (4) (2006) 046704.

[44] Z. Guo, C. Zheng, B. Shi, Lattice boltzmann equation with multiple effective relaxation times for gaseous microscale flow, Physical Review E 77 (3) (2008) 036707.

[45] Q. Li, Y. He, G. Tang, W. Tao, Lattice boltzmann modeling of microchannel flows in the transition flow regime, Microfluidics and nanofluidics 10 (3) (2011) 607-618.

[46] M. Levesque, O. Bénichou, B. Rotenberg, Molecular diffusion between walls with adsorption and desorption, The Journal of chemical physics 138 (3) (2013) 034107.

[47] J.-M. Vanson, F.-X. Coudert, B. Rotenberg, M. Levesque, C. Tardivat, M. Klotz, A. Boutin, Unexpected coupling between flow and adsorption in porous media, Soft matter 11 (30) (2015) 6125-6133.

[48] S. Ansumali, I. V. Karlin, Kinetic boundary conditions in the lattice boltzmann method, Physical Review E 66 (2) (2002) 026311

[49] Z. Guo, B. Shi, T.-S. Zhao, C. Zheng, Discrete effects on boundary conditions for the lattice boltzmann equation in simulating microscale gas flows, Physical Review E 76 (5) (2007) 056704.

[50] T. Inamuro, M. Yoshino, F. Ogino, A non-slip boundary condition for lattice boltzmann simulations, Physics of Fluids (1994-present) 7 (12) (1995) 2928-2930.

[51] C. Shen, D.-B. Tian, C. Xie, J. Fan, Examination of the $\mathrm{lbm}$ in simulation of microchannel flow in transitional regime, Microscale Thermophysical Engineering 8 (4) (2004) 423-432.

[52] T. Veltzke, J. Thöming, An analytically predictive model for moderately rarefied gas flow, Journal of Fluid Mechanics 698 (2012) 406-422.

[53] R. J. Ambrose, R. C. Hartman, M. Diaz-Campos, I. Y. Akkutlu, C. H. Sondergeld, Shale gas-in-place calculations part i: New pore-scale considerations, SPE Journal 17 (01) (2012) 219-229.

[54] G. Karniadakis, A. Beskok, N. Aluru, Microflows and nanoflows: fundamentals and simulation, Vol. 29, Springer, 2006.

[55] J. Sun, Z.-X. Li, Effect of gas adsorption on momentum accommodation coefficients in microgas flows using molecular dynamic simulations, Molecular Physics 106 (19) (2008) 2325-2332.

[56] M. Barisik, A. Beskok, Molecular free paths in nanoscale gas flows, Microfluidics and Nanofluidics 18 (5-6) (2015) 1365-1371.

[57] K. Wu, Z. Chen, X. Li, Real gas transport through nanopores of varying cross-section type and shape in shale gas reservoirs, Chemical Engineering Journal 281 (2015) 813-825.

[58] T. Yang, X. Li, D. Zhang, Quantitative dynamic analysis of gas desorption contribution to production in shale gas reservoirs, Journal of Unconventional Oil and Gas Resources 9 (2015) 18-30. 